\title{
Hyper-Redundant Manipulator Capable of Adjusting Its Non-Uniform Curvature with Discrete Stiffness Distribution
}

\author{
Seongil Kwon ${ }^{1,2}{ }^{\mathbb{D}}$, Jeongryul Kim ${ }^{3, *}$, Yonghwan Moon ${ }^{1,4}$ and Keri Kim ${ }^{1,2, *}$ \\ 1 Augmented Safety System with Intelligence Sensing and Tracking (ASSIST), Korea Institute of Science and \\ Technology, Seoul 02792, Korea; kstar1@kist.re.kr (S.K.); moonyh1230@kist.re.kr (Y.M.) \\ 2 Division of Bio-Medical Science and Technology, University of Science and Technology, Daejeon 34113, Korea \\ 3 Center for Healthcare Robotics, Korea Institute of Science and Technology, Seoul 02792, Korea \\ 4 School of Mechanical Engineering, Korea University, Seoul 02841, Korea \\ * Correspondence: jeongkim@kist.re.kr (J.K.); jazzpian@kist.re.kr (K.K.)
}

check for updates

Citation: Kwon, S.; Kim, J.; Moon, Y.; Kim, K. Hyper-Redundant Manipulator Capable of Adjusting Its Non-Uniform Curvature with Discrete Stiffness Distribution. Appl. Sci. 2022, 12, 482. https://doi.org/ 10.3390/app12010482

Academic Editor: Dario Richiedei

Received: 15 November 2021

Accepted: 29 December 2021

Published: 4 January 2022

Publisher's Note: MDPI stays neutral with regard to jurisdictional claims in published maps and institutional affiliations.

Copyright: (C) 2022 by the authors. Licensee MDPI, Basel, Switzerland. This article is an open access article distributed under the terms and conditions of the Creative Commons Attribution (CC BY) license (https:// creativecommons.org/licenses/by/ $4.0 /)$.

\begin{abstract}
Hyper-redundant manipulators are widely used in minimally invasive surgery because they can navigate through narrow passages in passive compliance with the human body. Although their stability and dexterity have been significantly improved over the years, we need manipulators that can bend with appropriate curvatures and adapt to complex environments. This paper proposes a design principle for a manipulator capable of adjusting its non-uniform curvature and predicting the bending shape. Rigid segments were serially stacked, and elastic fixtures in the form of flat springs were arranged between hinged-slide joint segments. A manipulator with a diameter of $4.5 \mathrm{~mm}$ and a length of $28 \mathrm{~mm}$ had been fabricated. A model was established to predict the bending shape through minimum potential energy theory, kinematics, and measured stiffnesses of the flat springs. A comparison of the simulation and experimental results indicated an average position error of $3.82 \%$ of the endpoints when compared to the total length. With this modification, the manipulator is expected to be widely used in various fields such as small endoscope systems and single-port robot systems.
\end{abstract}

Keywords: flexible robotics; kinematics; mechanism design; hyper-redundant manipulator; nonuniform curvature

\section{Introduction}

Minimally invasive surgery (MIS) is common medical practice owing to its convenience for surgeons and reduced patient trauma and recovery time [1-3]. The internal structure of the human body is diverse, and there are cases in which a surgical tool may have to pass through narrow and winding paths. In order to reach the target position and perform the necessary operations, we require surgical tools with flexible bodies [4]. As machines with flexible bodies and high dexterities, a considerable amount of research focuses on hyper-redundant manipulators [5-8]. Hyper-redundant manipulators used in MIS take an underactuated form that transmit power using tendons from the outside. This is advantageous for miniaturization and entering narrow and winding passages, due to its flexibility.

On the other hand, owing to the manipulator's underactuated characteristic, it is difficult to predict the shape and precisely follow the operator's intents, and determine the bending shape according to the situation and environment. Since the joint of the manipulator is a hyper-redundant structure, and the underactuated method controls all joints with one tendon, it is difficult to accurately predict the shape of the joint because it is not possible to control the motion of all joints individually. In addition, it is difficult to install the actuator for each joint in these manipulators, and since the module that transmits power is external and transmits power with tendons, it is difficult to perform precise 
control according to the environment. However, despite these difficulties to perform, precise control according to the environment and predicting are very important.

Predicting the manipulator's bending shapes helps plan various surgical routes with precise control [9-11]. To predict the curvature, some methods have utilized an elastic tube-shaped body, where the radius of curvature is assumed to be either constant, following Webster et al. [12] and Dalvand et al. [13], or variable, following T. Mahl et al. [14]. Other methods using kinematics and statics were introduced to analyze a manipulator with joints [15-18]. The principle of minimum potential energy for predicting the shape of the manipulator by Hwang et al. [19], applied in this study, is yet another powerful method, which has the advantage of being universally applicable.

Furthermore, a manipulator with a non-uniform curvature can provide the user with an appropriate option to select and apply a bending shape suitable for various working environments. Various attempts have been made to make non-uniform bending curvature of manipulators for particular circumstances. A continuum robot with a non-uniform curvature while making various bending shapes by changing the routing of the driving tendon was developed by Rucker et al. [20]. The active stiffness tuning method introduced by Kim et al. [21,22] inserted a coil spring into each joint to vary the curvature, but this required a separate drive for each joint. The manipulator proposed by Jiang et al. [23] consists of three different stiffness materials. It used heterogeneous soft materials that required reinjection molding to adjust the curvature. Although the bending part operated by one actuator has a uniform curvature, there are methods to connect several of them so that the overall curvature is non-uniform. The mechanism applied to decoupled joints proposed by Huang et al. [24] and the angular synchronous mechanism proposed by Qin et al. [25] were implemented to differentiate each bending sections and drive to make the overall bending shape non-uniform. The mechanism proposed by $\mathrm{Li}$ et al. [26] is also a suitable method to control the curvature using a constraint and bending part of the manipulator. Furthermore, Pana et al. [27] proposed a driving method using pneumatics and hydraulics instead of cables; this is another interesting method to achieve non-uniform curvature.

The main contribution of this study is the developed a hyper-redundant manipulator with elastic fixtures capable of predicting its bending shape, with non-uniform curvature. Additionally, this manipulator has two degrees of freedom (DoF) for bending motion, with a hollow through which surgical tools can pass, and a small diameter of $4.5 \mathrm{~mm}$. To predict the bending shape of manipulator, we applied the principle of minimum potential energy theory. This manipulator was fabricated by stacking small rigid segments serially. A hollow part in the middle of manipulator allowed the insertion of various surgical tools. The elastic fixtures, made in the form of flat springs, affected the proportion in which the joints move according to their stiffness. Different configurations of segments and elastic fixtures could achieve non-uniform curvatures of the manipulator's joints by the operator's intents. These non-uniform curvatures help make diverse bending shapes and workspaces, as shown in Figure 1.

In Section 2, the methods for the prediction of the manipulator's movement are discussed. Section 3 describes the prototype of the manipulator, its bending simulation and experimental evaluation. Section 4 discusses the results of this study, and Section 5 summarizes everything along with the conclusion. 


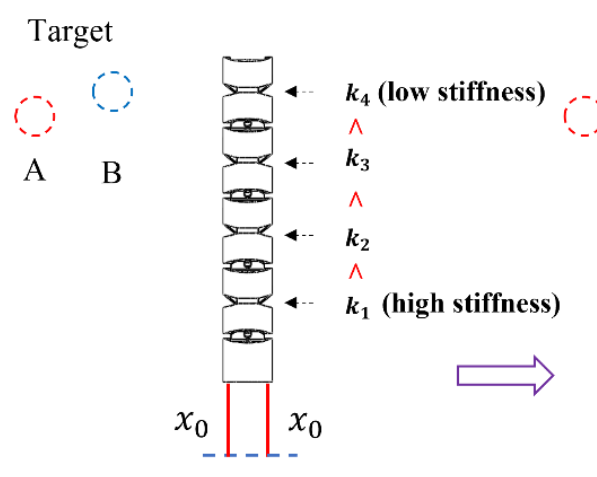

Target

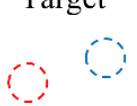

A B

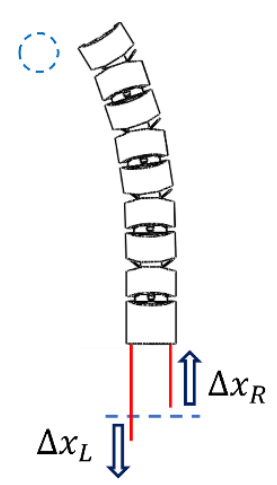

(a)

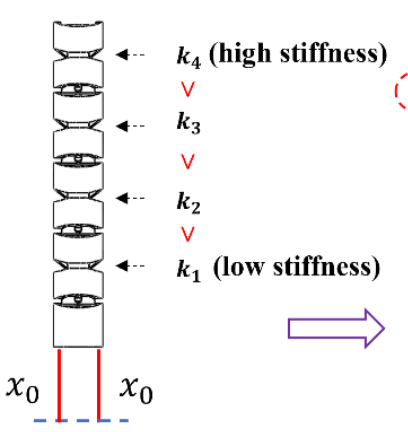

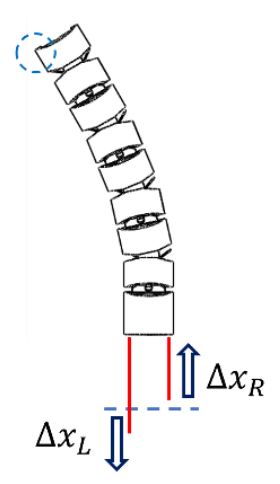
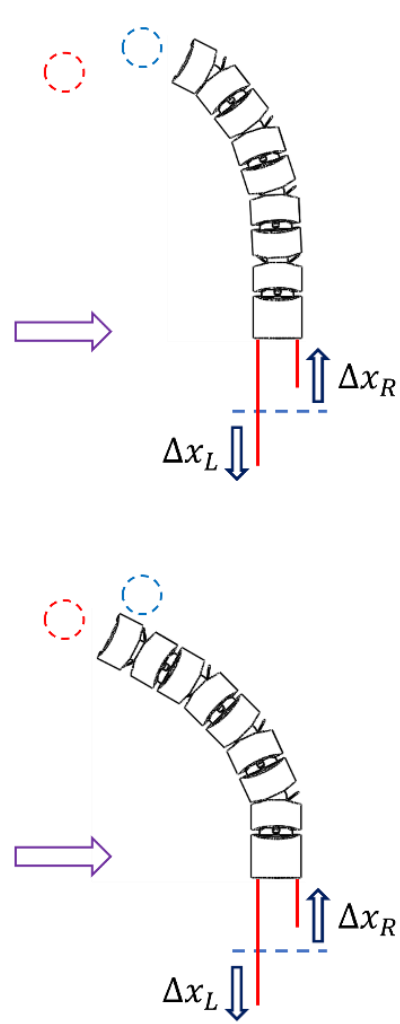

(b)

Figure 1. Examples of bending motion of a hyper-redundant manipulator with non-uniform curvature: (a) The curvature of the distal part is higher as it bends; (b)The curvature of the proximal part is higher.

\section{Method}

\subsection{Design and Modeling for Bending Shape Prediction of Flat Spring Mechanism}

To manufacture the hyper-redundant manipulator, the segments forming each joint were designed. The specifications of the manipulator targeted in this study are as follows: a 2-DoF bending motion should be possible to realize intuitive movement; elastic fixtures should be placed in the middle of segments; there must be a hollow through which surgical tools can pass. We achieved an optimal design to reduce the diameter of the manipulator for application in MIS. Figure 2a shows one joint of the manipulator, comprising an upper and a lower segment. The joint of the manipulator consists of a protrusion in the upper segment and an indentation in the lower segment. The indentation and protrusion were twisted by $90^{\circ}$. They allowed the manipulator to have two bending directions. A flat spring with a special shape was designed and adopted for the elastic fixture placed at each joint of the manipulator. When placing the elastic fixture on the joint of the manipulator, the position and fixation method can be tried diversely. A typical coil spring or a superelastic rod can be used $[28,29]$. However, the coil spring arrangement would not help minimize the manipulator's diameter. It would cause friction by colliding with the cable, which became an obstacle to the operation. Even if the rod-shaped elastic fixtures are suitable enough with thin and simple design, sometimes cause hardship on fixing methods or limit degrees of freedom. In this study, we proposed a special shape of flat spring to meet the aforementioned design requirements. Figure $2 \mathrm{~b}$ shows a flat spring, which functioned as an elastic fixture between segments. It has a hollow in the middle and does not interfere with cable movement and two bending movements. The wings on both sides maintained balance when the segments were assembled serially. Figure 2c shows the assembly of the segment and the flat spring. 


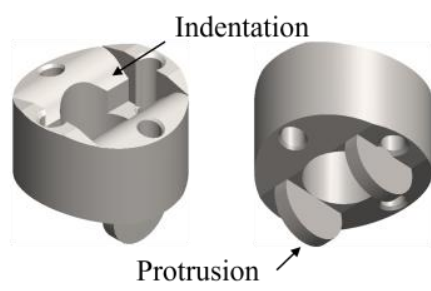

(a)

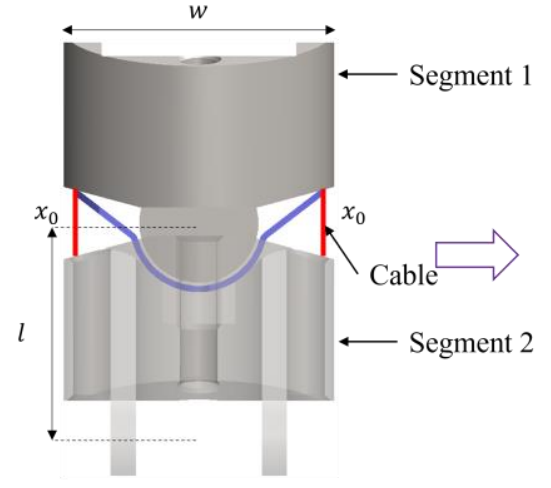

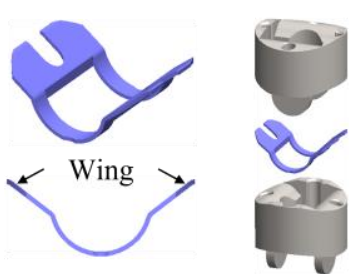

(c)

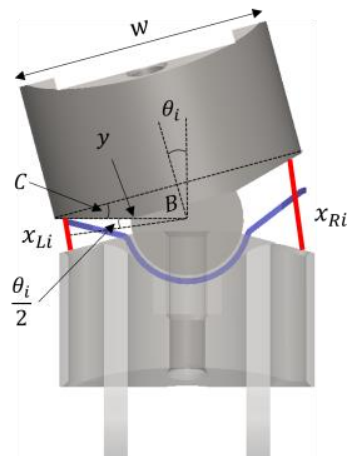

(d)

Figure 2. Arrangement of the flat spring between the manipulator's joints; (a) is a segment of the manipulator, (b) is a specially designed flat spring, and (c) is a joint assembly that has two segments and one flat spring. (d) Initially, they were aligned in a line (left panel). When the cable was pulled, the angle of bending varied depending on the stiffness of the flat spring (right panel).

The left panel of Figure $2 \mathrm{~d}$ shows the initial position when two segments and a flat spring were assembled, and the right panel shows the state when it was bent. Looking at the bending state, if we know the length $x_{L i}$, of the cable after being pulled, we can find the bending angle $\theta_{i}$, as follows:

$$
\theta_{i}=2 \cdot \sin ^{-1}\left(\frac{x_{L i}}{2 y}\right), \quad y=\sqrt{B^{2}+\left(\frac{w}{2}\right)^{2}} .
$$

where $y$ denotes the length of the hypotenuse cut from the center of rotation of the joint, to the outside of the segment. $B$ denotes the height from the start of the inclined plane to the axis of rotation. Additionally, $w$ denotes the width of the segment. The angle of inclined plane could be obtained from $B$ and $w$.

Fundamentally, when an external force is applied to an elastic object to move the object, the work done at that time is stored as energy in the object. The theory, known as Hooke's law, can be applied to all elastic bodies, including helical coil springs. This theory can also be applied to the case of the manipulator. Most manipulators fix one end-part of the cable at the end of the manipulator and pull and release the cable for a bending motion. A typical manipulator assumes no resistance when pulling the cable. However, as suggested in this study, when an elastic fixture was placed in the joint, a resistance force was generated. Given the pulling force, the length of the cable, and the elastic modulus of the body, the stored energy $E_{S}$ can be calculated as:

$$
W=\int_{x_{0}}^{x_{e}} k_{t} x d x=\frac{1}{2} k_{t} x_{e}^{2}=E_{s}
$$

where $x$ is the displacement of the cable from initial position $\left(x_{0}=0\right)$ to pulled position $x_{e}$, and $k_{t}$ is the total elastic modulus.

The stored energy equation for one joint can be expressed as given in (2), but the manipulator developed in this study had multiple joints. Another important feature was that flat springs with different thicknesses were placed at each joint. Different thicknesses of 
these flat springs meant that they had different modulus, which influenced the stiffness. The total moved cable displacement could be expressed as the sum of the cable displacements moved at each joint, and the total energy formula could be derived by using the stiffnesses of the flat spring placed at each joint.

The cable displacement, $\Delta x_{i}$, of each joint is expressed as $x_{\text {initial }}-x_{L i}$, the result of subtracting the cable length, $x_{L i}$, after bending, from the initial cable length, $x_{\text {initial }}$. When the number of joints was increased to $n$, the displacement $\Delta x_{i}$ of the cable moved by compressing the elastic fixture at each joint and the total displacement of the pulled cable $x_{e}$ could be expressed as follows:

$$
x_{e}=\Delta x_{1}+\cdots+\Delta x_{n}
$$

Using Formulas (2) and (3), the total energy stored when driving a manipulator with $n$ joints can be calculated as follows:

$$
E_{S}\left(x_{e}\right)=\frac{1}{2}\left(k_{1} \Delta x_{1}^{2}+\cdots+k_{n} \Delta x_{n}^{2}\right)=\frac{1}{2} \sum_{i=1}^{n} k_{i} x_{i}^{2} .
$$

The total energy is the sum of the energies of each joint and the energy of each joint consists of the product of the cable displacement moved at each joint and the stiffness of the flat spring. The cable length for each joint and bending shape of the manipulator is determined by minimizing the energy stored, according to the minimum potential energy theory. Conversely, when the lowest possible stored energy was known, the cable lengths of each joint could be derived, and then the bending shape of the entire manipulator could be predicted.

If the stiffness of the flat springs placed on each joint is the same, the overall stiffness can be easily calculated even if the number of joints increases. If the stiffness of the flat springs placed at each joint is different, the formula for deriving the overall stiffness becomes somewhat complicated as the number of joints increases. Moreover, this is only possible under the assumption that stiffness is constant. However, the shape of the flat spring proposed in this study shown in Figure $2 \mathrm{~b}$ was bizarre and had a different shape from the general helical coil spring. As a result, the stiffness of the flat spring is expressed in the form of a function rather than a constant. This can be obtained through experimentation, and calculated using the following equation using the compressed displacement and the applied external force:

$$
k_{j}(\delta x)=\frac{F_{e x t}(\delta x)}{\delta x}, j=0.08,0.1,0.12,0.14
$$

where $k_{j}$ is the stiffness calculated, and $j$ represents thickness of each flat spring with mm unit, $\delta x$ is the displacement compressed by an external force, and $F_{\text {ext }}$ is the external force. The stiffness value in the form of a function complicated the calculation to obtain the total minimum energy value. We applied constrained optimization to solve this complex computation. The ultimate goal was to find the minimum value of energy calculated using the cable displacement $\Delta x_{i}$ by compression of each joint and the stiffness $k_{j}$ at that time, which can be expressed as:

$$
\underset{k_{j}, \Delta x_{i}}{\arg \min }\left(E_{S}\left(x_{e}\right)\right) .
$$

At this time, it should be considered that there is a distance that can be maximally compressed according to the thickness of the flat spring. This is expressed as $l_{m d}$, and this value could also be obtained through experiments. The experiment to calculate the stiffness of the flat spring and the experiment to measure $l_{m d}$ will be dealt with in section III. 


\subsection{Kinematic Analysis and Prediction Process}

We can acquire an endpoint of the manipulator using forward kinematics. Figure 3 shows the shape of the manipulator with arbitrary bending angles and parameters of the segments. The forward kinematics were solved using the Denavit-Hartenberg (D-H) parameters. The parameters applied in this method are showed in Table 1 . Where the origin of the absolute coordinate system is $o(0,0,0)$, the position of the manipulator endpoint ${ }^{0} P\left(x_{e}, y_{e}, z_{e}\right)$ in the coordinate system is expressed as

$$
{ }^{0} P={ }_{1}^{0} T \cdot{ }_{8}^{7} T \cdot{ }^{e} P={ }_{8}^{0} T\left[\begin{array}{c}
l_{e} \\
0 \\
0 \\
1
\end{array}\right] .
$$

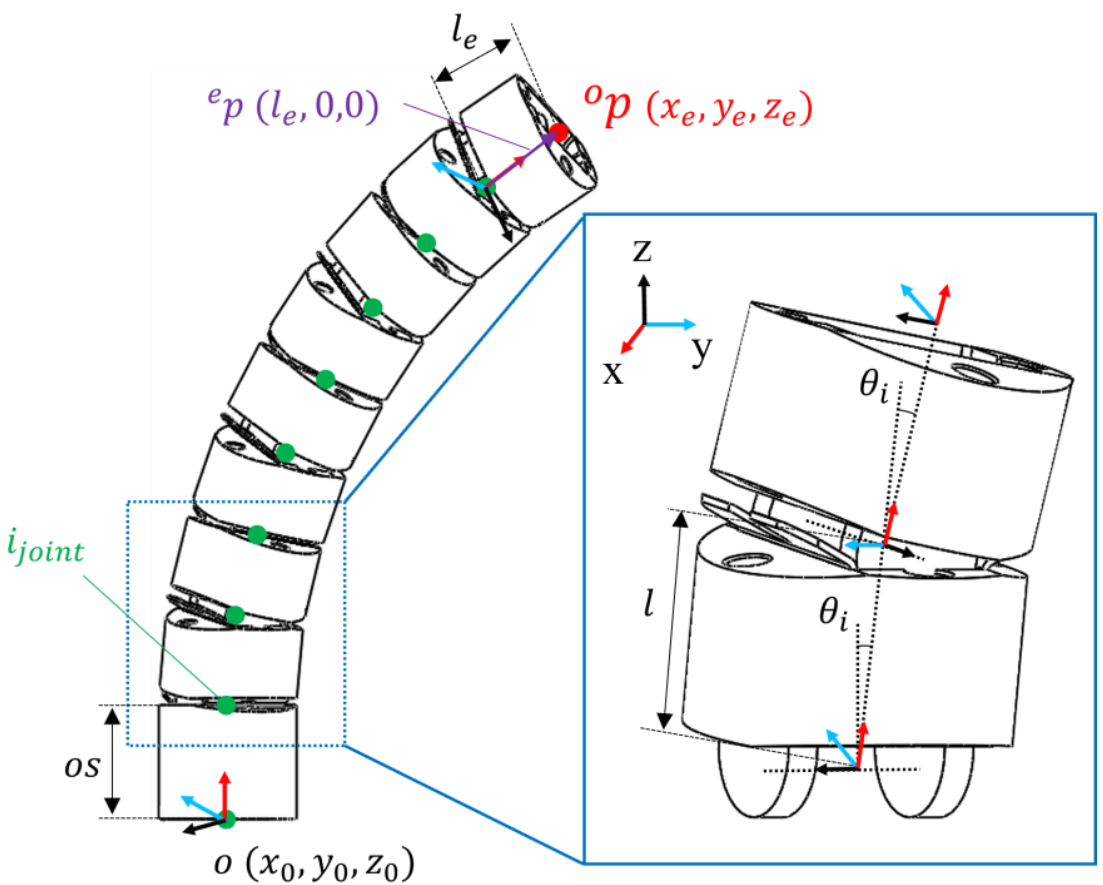

Figure 3. Coordinate system setting for kinematic analysis and explanation of each parameter.

Table 1. Denavit-Hartenberg (D-H) parameters.

\begin{tabular}{ccccc}
\hline $\boldsymbol{i}$ & $\boldsymbol{d}_{\boldsymbol{i}}$ & $\boldsymbol{\theta}_{\boldsymbol{i}}$ & $\boldsymbol{\alpha}_{\boldsymbol{i}-\mathbf{1}}$ & $\boldsymbol{r}_{\boldsymbol{i}-\mathbf{1}}$ \\
\hline 1 & 0 & $\theta_{1}$ & 0 & os \\
2 & 0 & $\theta_{2}$ & $90^{\circ}$ & $l$ \\
3 & 0 & $\theta_{3}$ & $-90^{\circ}$ & $l$ \\
4 & 0 & $\theta_{4}$ & $90^{\circ}$ & $l$ \\
5 & 0 & $\theta_{5}$ & $-90^{\circ}$ & $l$ \\
6 & 0 & $\theta_{6}$ & $90^{\circ}$ & $l$ \\
7 & 0 & $\theta_{7}$ & $-90^{\circ}$ & $l$ \\
8 & 0 & $\theta_{8}$ & $90^{\circ}$ & $l$ \\
\hline
\end{tabular}

${ }_{1}^{0} \mathrm{~T}$ is a transformation matrix representing the relationship between the origin and the first joint's coordinate systems and can be expressed as ${ }_{8}^{0} \mathrm{~T}$ by extending the relationship from the origin to the eighth joint. ${ }^{e} P$ represents the endpoint with respect to the last relative coordinate system. It can be represented by ${ }^{e} P\left(l_{e}, 0,0\right)$ because the endpoint is located on the x-axis. The value of os in Figure 3, which corresponds to the column $r_{i-1}$ of the first joint, and the value $l_{e}$ on the matrix ${ }^{e} P$ could be changed depending on the length of the segment assembled at the proximal and distal parts. 
The overall flow chart for predicting the bending motion of the manipulator by applying the results of the kinematic analysis is shown in Figure 4. When the cable was pulled a certain distance, the cable displacement of each joint with the minimum potential energy could be known through constraint optimization, and the shape of the manipulator could be predicted using this.

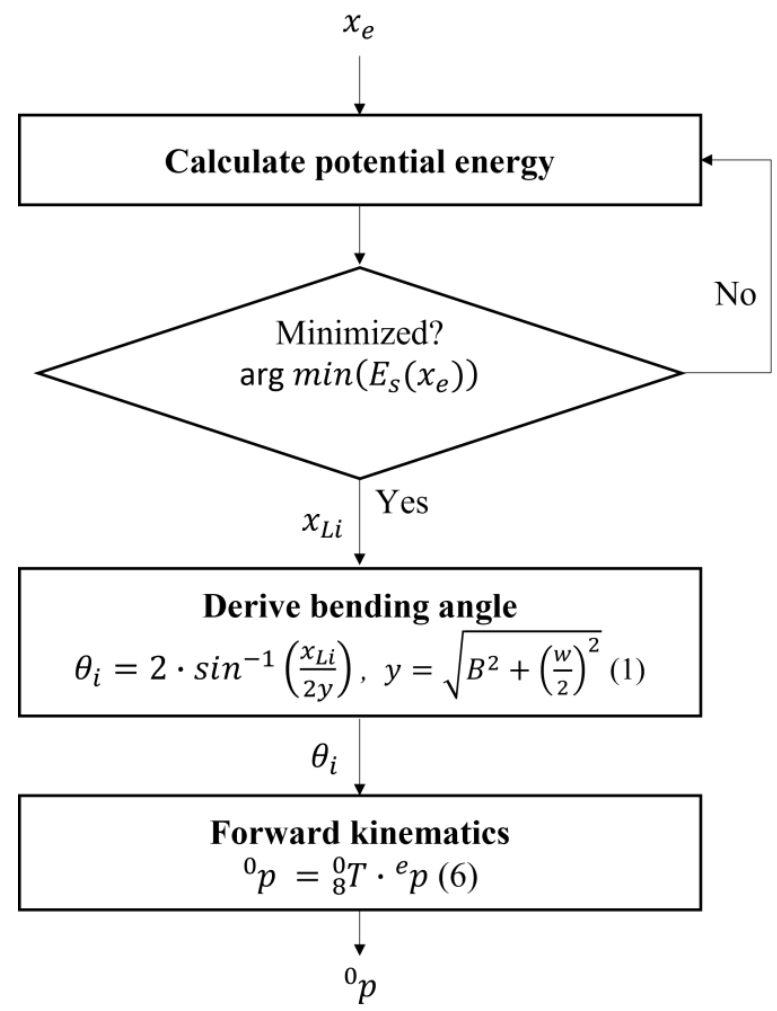

Figure 4. Flow chart for predicting the bending motion of hyper-redundant manipulator with non-uniform curvature and formulas applied in each step.

\section{Evaluation}

\subsection{Flat Spring Stiffness Function}

The curvature of the manipulator proposed in this study was significantly influenced by the characteristics of the flat springs placed between the segments. These flat springs had a complex shape. Therefore, their spring coefficients are nonlinear as opposed to constant values in the case of a general helical spring [30]. The stiffness values of these flat springs could be obtained using an analysis program. However, owing to their complex shape, the stiffness values were obtained through experiments instead. The left panel in Figure 5a schematically shows the direction in which the flat spring received the force when the segment of the manipulator was bent. Stiffnesses could be obtained using Equation (5) and the set. The image of the compressed flat springs was captured using a telecentric lens (Edmund, United States) with the distance being calculated in pixels. Flat springs with the following four different thicknesses were tested: $0.08,0.1,0.12$, and 0.14 (mm). Figure 6 shows the experimental results; the relationship between the compressed distance and force based on the initial position is expressed as stiffness, and the maximum compression distance $l_{m d}$ for each thickness was measured to be $0.68,0.58,0.33,0.22(\mathrm{~mm})$ when the thicknesses were $0.08,0.1,0.12$, and $0.14(\mathrm{~mm})$, respectively. It was confirmed that as the pulled distance increased, the stiffness increased owing to the restriction posed by the segment shape or strong resistance. The distance data, from 0.2 to $1.0 \mathrm{~mm}$, were extracted and a trend line in the form of a linear function was obtained by linear regression. The data were applied to the curvature prediction simulation. The trend line function for each thickness can be seen in the top left panel of Figure 6. Through an experiment, the stiffness 
function $k_{j}$ based on the thickness of the flat spring was obtained. All lines had a positive slope; the greater the thickness, the higher the y-intercept value (initial stiffness).

The shape of a flat spring with a thickness of $0.1 \mathrm{~mm}$ is shown in the right panel in Figure $5 \mathrm{a}$. First, the design of the flat spring to be manufactured was traced on the thin metal plate, which was then cut. The cut metal plate was held between a jig for molding by compression and heat. The manufactured flat springs (SUS 302-material used for general springs with high rigidity) were placed between two segments and fixed by an adhesive to the indentation of the lower segment. After applying $100 \mathrm{~g}$ pre-tension using the driving part, the cables were pulled and released in steps of $0.1 \mathrm{~mm}$ to bend the segment.

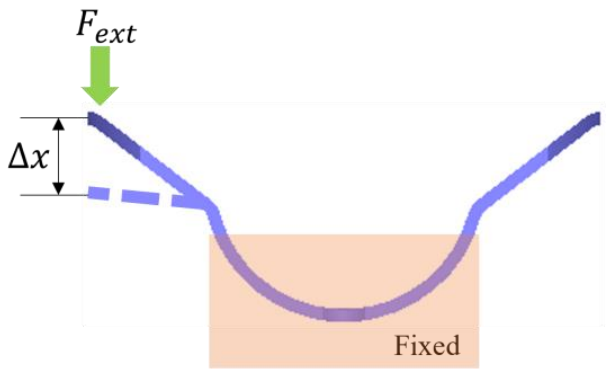

(a)

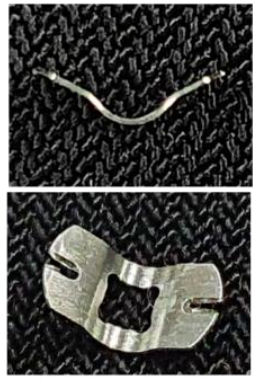

Flat spring

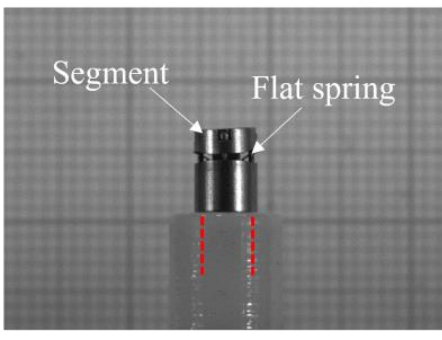

Initial shape

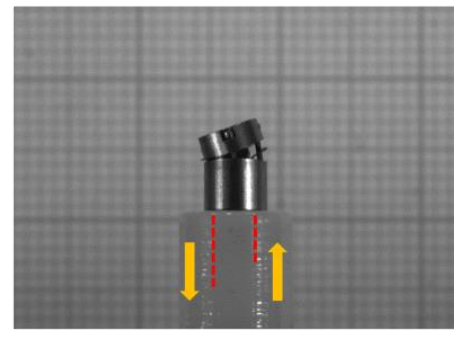

(b)
Maximally bent

Figure 5. Schematic diagram of external force and displacement measured to calculate stiffness and a fabricated flat spring; (a) Actual experiment to measure external force and displacement; (b) Fabricated flat spring.

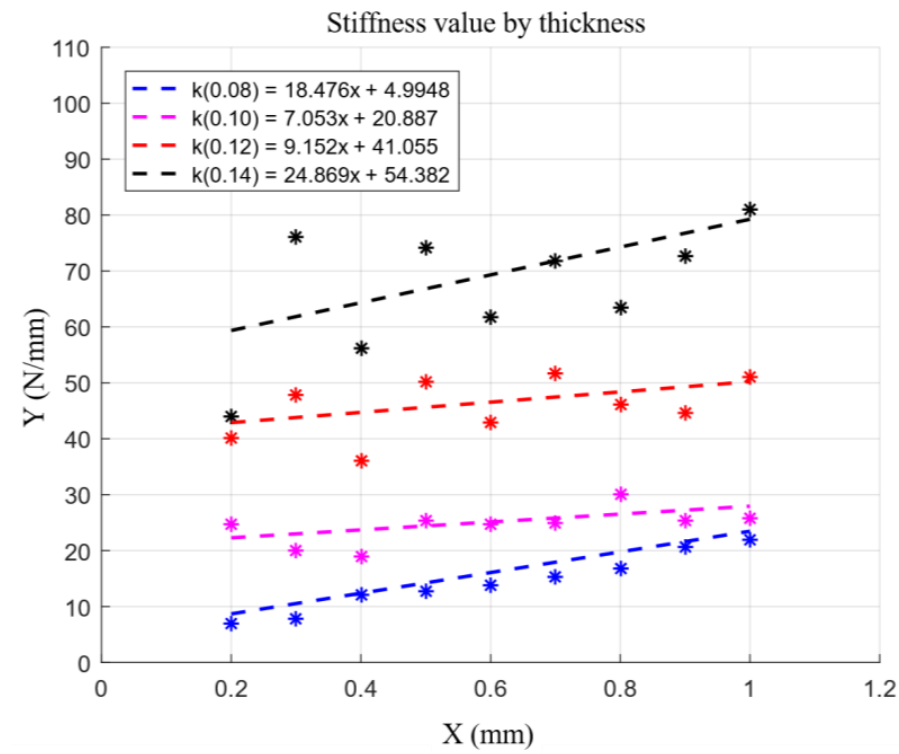

Figure 6. Stiffness graph and linearized trend of the flat springs obtained experimentally. 


\subsection{Prototype}

A prototype was machined and assembled to conduct the experiment, as shown in Figure 7. The manipulator consisted of seven middle parts, one distal part, and one proximal part (SUS 304), and the flat springs were placed at each joint. A circular indentation and a protrusion were present at the top and bottom of the middle part; the radius of curvature $\left(r_{p}=0.95 \mathrm{~mm}\right)$ of the protrusion was fixed, but the radius of curvature $\left(r_{d}=0.98\right.$, $1.0,1.1,1.2 \mathrm{~mm}$ ) of the indentation was machined by adjusting the length according to the flat spring used. The angle $C$ of the inclined surface was $10.8^{\circ}$, which indicated a bending of $21.6^{\circ}$ per joint (the actual bending was slightly less than this value owing to the resistance of the flat spring). The outer diameter $w$ of the segment was $4.5 \mathrm{~mm}$; the segment was hollow $(c h=2 \mathrm{~mm})$, with four side holes $(s h=0.6 \mathrm{~mm})$. The cable for driving the manipulator passed through the side hole. The center hole could be used by the operator either for transmitting force, such as forceps or scissors, or for passing energy to the site in the form of electrodes or lasers. The proximal part had a small groove for coupling with another part, and taking this into account, the total length of the manipulator was approximately $28 \mathrm{~mm}$. Sophistication in the manufacturing process is the best approach to reduce the error between actual bending experimental and simulation results. Even a slight distortion, when fixing the segments and flat springs, can lead to a significant error. Hence, the assembly should be given a careful consideration. When assembling this prototype, the flat spring was applied with adhesive and turned upside down to maintain the balance, and the segment was then placed on it.

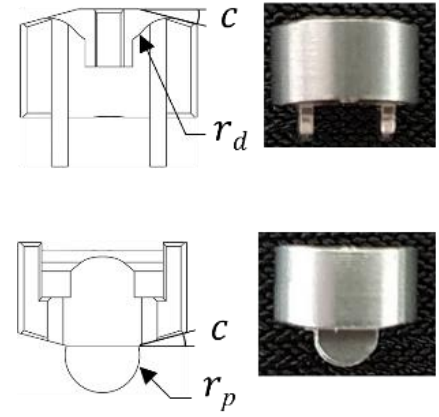

(a)

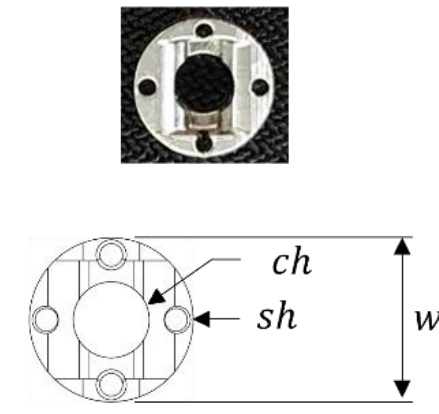

(b)

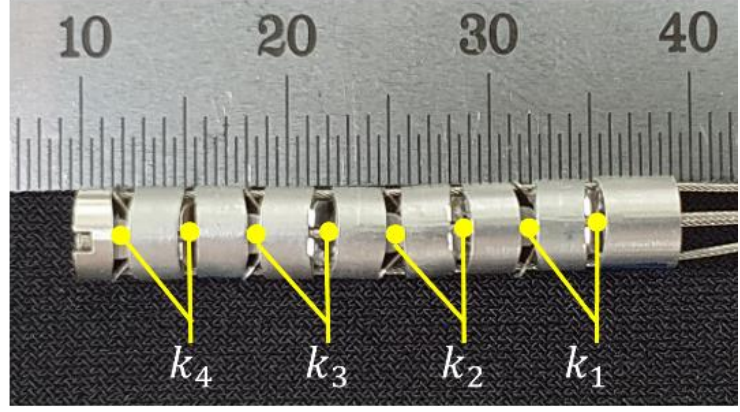

(c)

Figure 7. Design of transmittance and prototype of the middle segment: (a) The side view; (b) The top view; (c) A prototype fabricated by assembling all segments and flat springs.

\subsection{Simulation}

Previously, the method of predicting the bending shape of the manipulator by applying the minimum potential energy theory was described. In addition, stiffness, which is a parameter necessary for prediction, and the movement distance of a cable whose movement was restricted due to physical limitations, were obtained through an experiment. As we mentioned earlier, it is complicated to derive the minimum energy value when the manipulator is bent. An optimizer was used to solve this complex equation. The MATLAB optimization function "fmincon" was used to consider all cases. The algorithm used was "sequential quadratic programming (SQP)". The total pulled distance of the cable was increased in units of $0.01 \mathrm{~mm}$. Following this, the minimum of the potential energy was identified by changing the distributed length of each joint. Figure 8 a shows the results of the simulation conducted with different thicknesses of flat springs placed at each joint. The bending shape of the manipulator depended on the combination of flat springs used for each joint. Additionally, the displacement that the cable can move was determined according to the flat spring assembled to the joint. The order of the thickness of the flat springs assembled from the proximal-part joint to the distal-part joint of the manipulator is shown in Figure 8b. The configuration $\mathrm{T} 1$, the red text, has a $0.08 \mathrm{~mm}$ thick flat spring on the proximal joint 
and a $0.14 \mathrm{~mm}$ thick flat spring on the distal part. Maximizing the difference between the thicknesses of the flat springs of the proximal joint and the distal joint could help maximize the difference in the curvature of the manipulator. Among the various configurations, the four selected flat spring configurations that could increase the difference in curvature were simulated. For each manipulator, the cable was pulled in steps of $0.5 \mathrm{~mm}$ each to achieve a displacement of $2.0 \mathrm{~mm}$ diagonally ( $y$-axis and $z$-axis). The configuration of flat springs marked in blue and green, and those marked in red and black appeared to bend with a similar tendency when the cable is pulled slightly.

\section{$0.5 \mathrm{~mm}$}

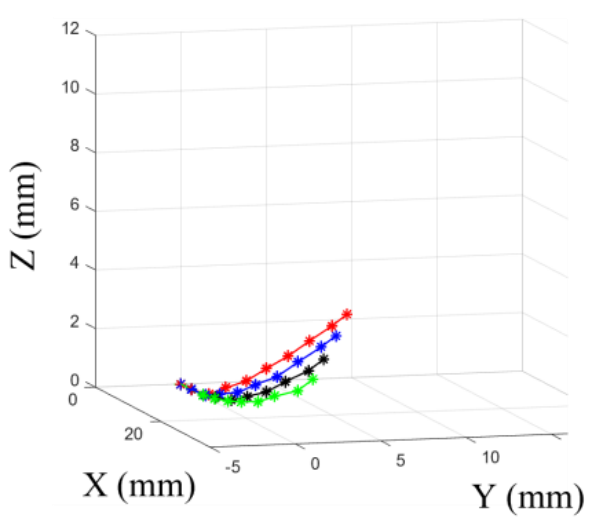

\section{$1.5 \mathrm{~mm}$}

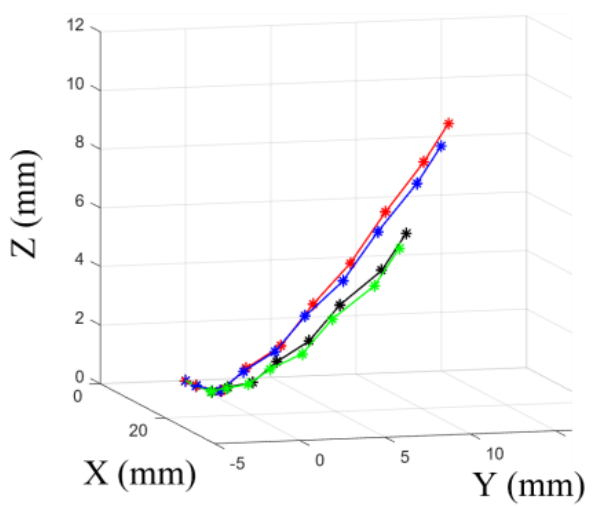

$1.0 \mathrm{~mm}$

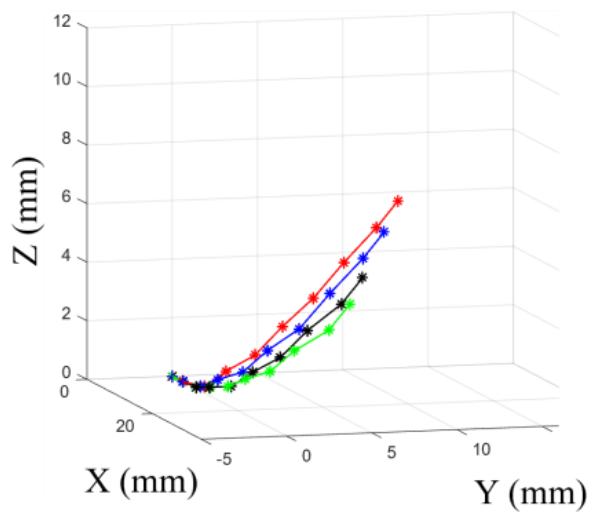

$2.0 \mathrm{~mm}$

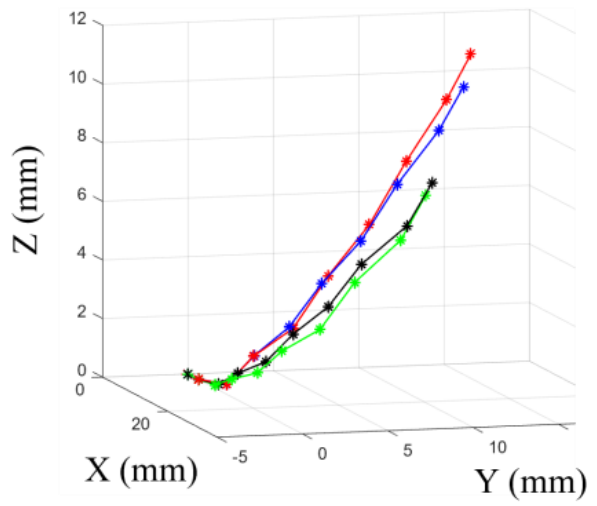

(a)

\begin{tabular}{|l|l|l|l|}
\hline Test(color) & \multicolumn{3}{|c|}{ The order of thickness of flat springs (mm) } \\
\hline T1(Red) & $0.08,0.10,0.12,0.14$ & T3(Blue) & $0.14,0.1,0.1,0.1$ \\
\hline T2(Green) & $0.14,0.12,0.10,0.08$ & T4(Black) & $0.1,0.1,0.1,0.14$ \\
\hline
\end{tabular}

(b)

Figure 8. Manipulator bending shape according to four flat springs combinations (a) Simulation result of pulling the driving cables of four different manipulators in $0.5 \mathrm{~mm}$ increments up to $2 \mathrm{~mm}$; (b) Indication by color what thickness of flat springs each manipulator is composed of. 


\subsection{Experiments}

Several experiments were conducted on the prototype manipulator to compare simulations with actual bending shapes. Figure 9 shows the experimental setup to obtain the stiffness values of the flat springs. Four motors (Maxon, Swiss) and linear actuators were arranged radially in a drive system. A load cell was connected to the cable (SUS 304, Carl Stahl Sava Industries, Riverdale, NJ, USA) driving the manipulator, enabling the tension applied to the cable to be measured. First, we checked if the bending curvature of the manipulator changed when configurations of flat springs with different thickness, arranged at the joint, were changed. To intuitively analyze the movement of the manipulator, it was observed on a plane surface. Figure 10a shows the result of the configuration of T1 and Figure $10 \mathrm{~b}$ shows the configuration of $\mathrm{T} 2$ of the manipulator. The operating cable of the manipulator of the two configurations (T1, T2) was pulled by $0.5,1.0,1.5$, and $2.0(\mathrm{~mm})$. It was confirmed that the proximal part in the case of T1, and the distal part in the case of $\mathrm{T} 2$, had a small radius of curvature about $4.27 \mathrm{~mm}$ when it maximally bent. Additionally, the distal part in the case of $\mathrm{T} 1$ and the proximal part in the case of $\mathrm{T} 2 \mathrm{had}$ a small radius of curvature about $14.67 \mathrm{~mm}$. The joint configuration of T1 had a larger workspace than T2. Next, the similarity between the simulations and the experiments was confirmed. To check for the similarities, the simulation results were superimposed on the manipulator of the actual experiments in Figure 11. The experimental values and the position errors against the simulation are shown in Table 2. In both combinations, the position error of the endpoint increases as the cable displacement increases.

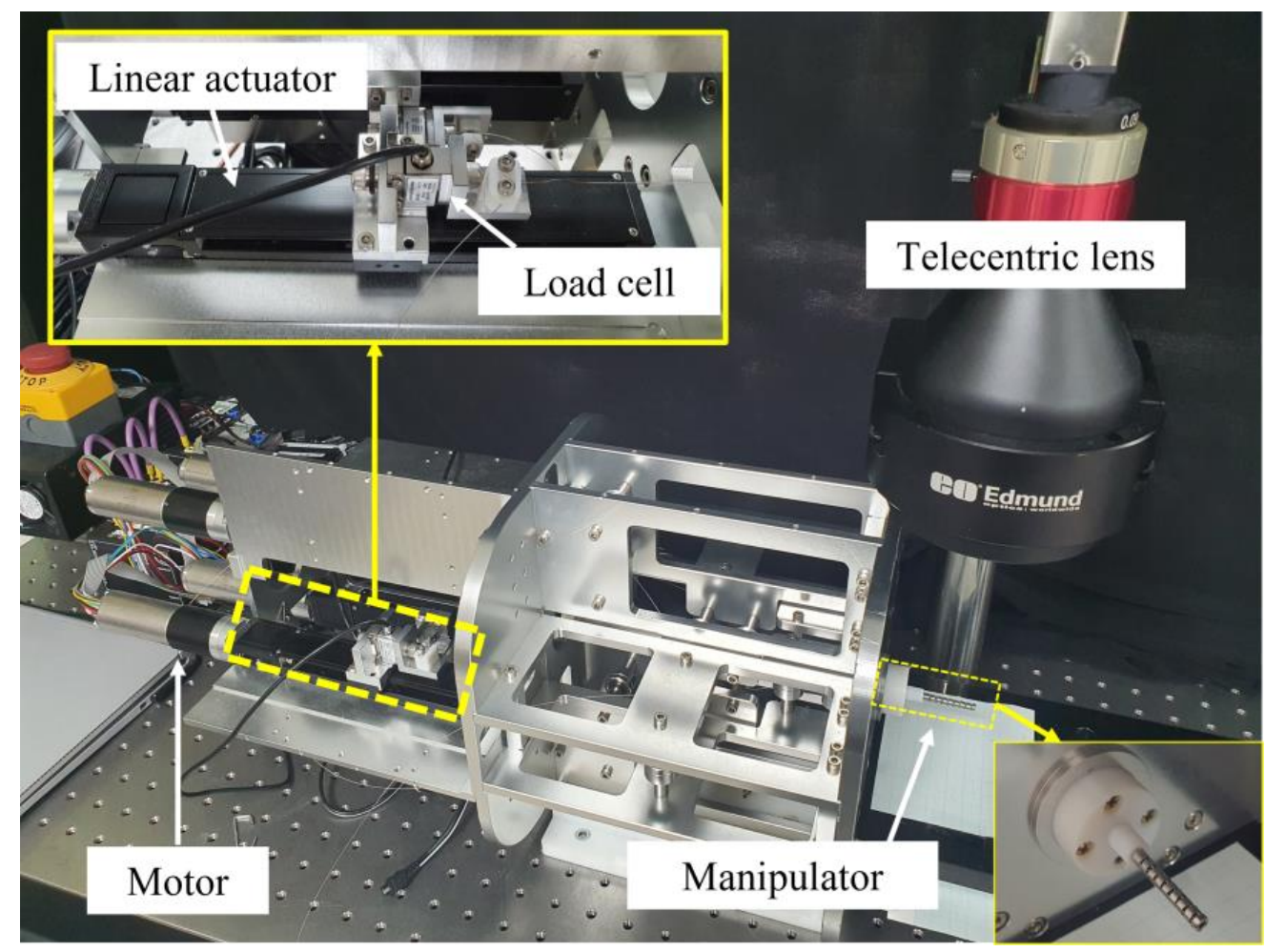

Figure 9. Experimental settings for manipulator's bending motion. The driving cable is moved using a linear actuator with a fixed load cell. After connecting the manipulator, as shown in the lower right picture, the movement is observed from the top vertically using a telecentric lens and a camera. 


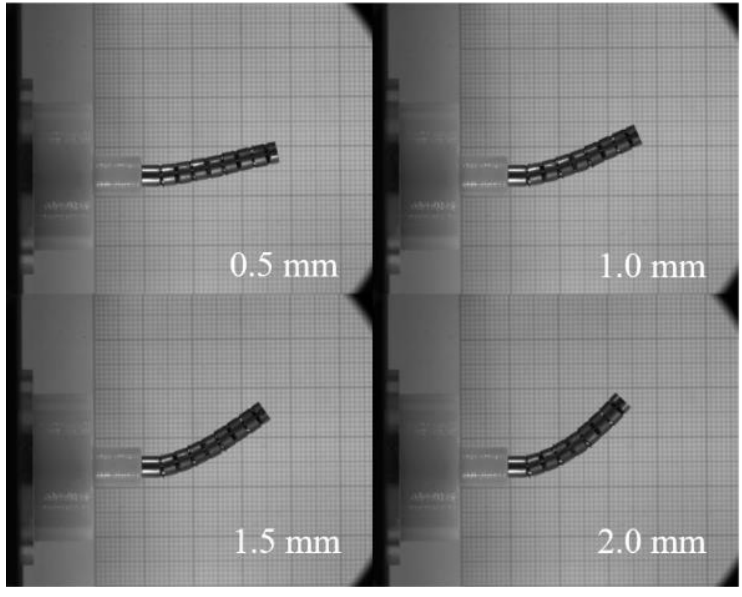

(a)

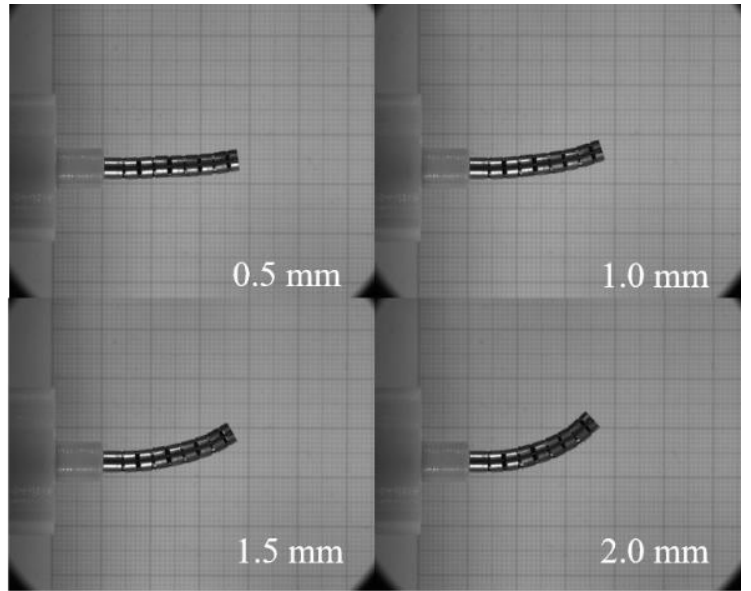

(b)

Figure 10. Results of manipulator bending experiment of two combinations; (a) Experimental result of T1 (red line) combination manipulator, moving the cable up to $2 \mathrm{~mm}$ at $0.5 \mathrm{~mm}$ intervals; (b) Experimental result of T2 (green line) combination manipulator performed under the same conditions.

T1
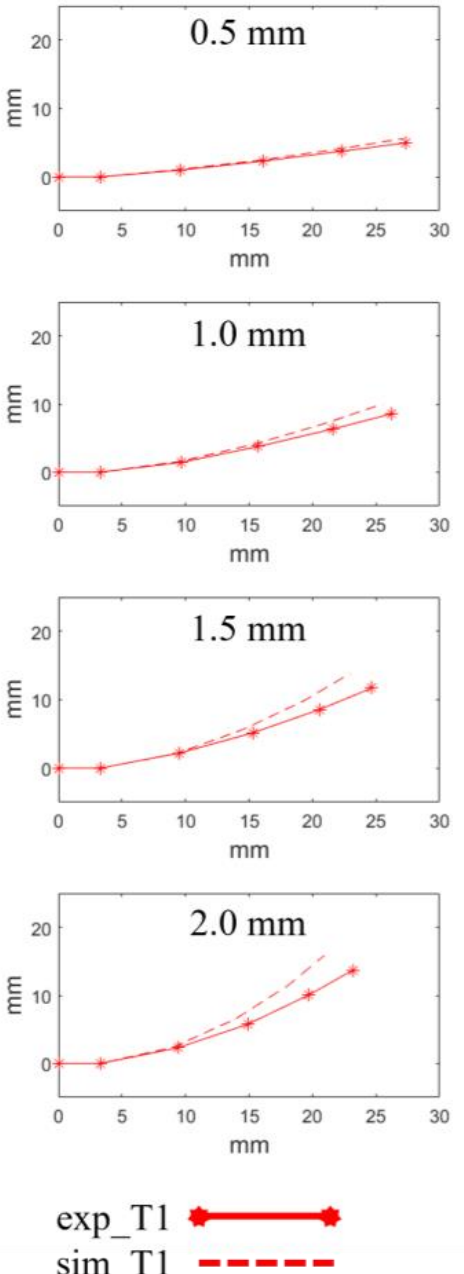

\section{$\mathrm{T} 2$}
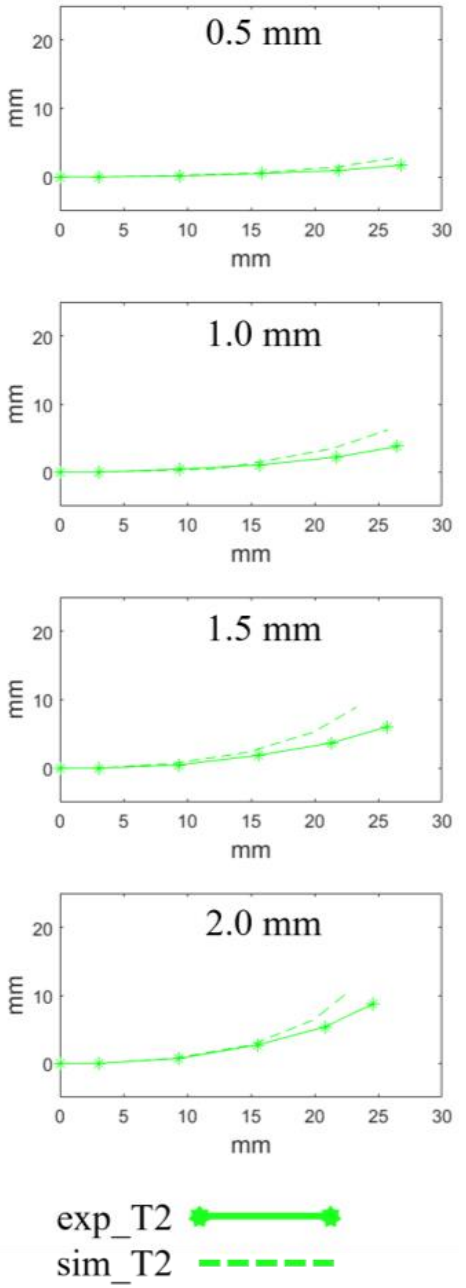

Figure 11. Graphs comparing experimental results and simulations. The two combinations (T1, T2) were compared, respectively, and the solid line represents the experimental data, and the dotted line represents the simulation data. 
Table 2. Position error of each joint.

\begin{tabular}{cccccc}
\hline \multirow{2}{*}{ Config. } & $\begin{array}{c}\text { Pulling } \\
\text { Length }(\mathbf{m m})\end{array}$ & \multicolumn{4}{c}{ Difference of ith Joint and Endpoint (mm) } \\
\cline { 3 - 6 } & 0.5 & 2nd & 3rd & 4th & Endpoint \\
\hline \multirow{3}{*}{$\mathrm{T} 1$} & 1.0 & 0.11 & 0.26 & 0.39 & 0.66 \\
& 1.5 & 0.19 & 0.49 & 1.02 & 1.61 \\
& 2.0 & 0.20 & 0.92 & 1.78 & 2.73 \\
$\mathrm{~T}$ & 0.5 & 0.09 & 0.99 & 4.62 & 2.27 \\
\hline \multirow{2}{*}{$\mathrm{T} 2$} & 1.0 & 0.14 & 0.43 & 0.53 & 1.18 \\
& 1.5 & 0.47 & 0.99 & 2.30 & 2.54 \\
& 2.0 & 0.26 & 0.56 & 1.38 & 3.75 \\
& & & & & 2.66 \\
\hline
\end{tabular}

\section{Discussion}

In this study, we introduced a hyper-redundant manipulator with elastic fixtures capable of predicting its bending shape with non-uniform curvature for MIS. A specially manufactured flat spring was placed between the hinged-slide joints to alter the non-uniform curvature by reassembling. The experimental results confirmed that the manipulator could have various non-uniform curvatures, by changing the configuration of the flat spring. The manipulator manufactured in this study had four joints, and the number of combinations of flat springs that can be arranged for each joint was quite large. Among them, simulations were performed on four combinations, and experiments were performed on two combinations for comparison. The experimental results confirmed a similar bending shape tendency when analyzed in simulation, proving that the applied minimum energy theory is established. Manipulators with different non-uniform curvatures according to different combinations had various workspaces. It was confirmed that the two combinations of manipulators in the experiment have different workspaces, which could allow the user to choose according to a specific environment or surgical operation.

The combination showing the most remarkable difference of the manipulator's bending shape in the simulation was T1 and T2. The experimental result of Figure 11 also showed the same trend as the simulation, and the shape difference between the two combinations was the largest when the final pulled distance was $2 \mathrm{~mm}$. This could be confirmed by the different positions of the joints having the minimum radius of curvature. In the case of the $\mathrm{T} 1$ combination, the proximal part had a minimum radius of curvature, and in the T2 combination, the distal part had a minimum radius of curvature. This affected the final position of the endpoints. In the T1 combination, the endpoint was located on the positions $23.19 \mathrm{~mm}$ and $13.71 \mathrm{~mm}$, on the horizontal and vertical axes, respectively. Additionally, in the T2 combination, the endpoint was located on the positions $24.57 \mathrm{~mm}$ and $8.76 \mathrm{~mm}$, on the horizontal and vertical axes, respectively. The two positions showed a difference of $5.14 \mathrm{~mm}$, which was quite significant, given that the total length of the manipulator is $28 \mathrm{~mm}$. Hence, it could be said that it had a great influence even when it was applied to a surgical target with a small working space.

The bending shape also had a reasonably high prediction rate. After matching the starting point and the first joint, the position errors from the second joint to the last joint were measured. As a result, the average error rate of the $\mathrm{T} 1$ combination was $3.48 \%$, the average of the T2 combination was $4.16 \%$, and the overall average error rate was $3.82 \%$. It could be confirmed that the bending shape analyzed in terms of the minimum energy was quite effective.

However, despite having a low average error rate, it could be confirmed that the maximum error was when the cable was pulled to the maximum of $2 \mathrm{~mm}$. When comparing the experiment and simulation, an error of up to $3.75 \mathrm{~mm}$ occurred at the endpoint of the manipulator of the $\mathrm{T} 2$ combination, which was about $13.4 \%$ when considering the total length of $28 \mathrm{~mm}$. 
There were multiple sources of errors in our study. Errors in manufacturing, buckling problems that occur when setting the initial position, deriving stiffness through linearization, plastic deformation of flat springs, and friction can be considered as error factors. Among these, the buckling problem is a common problem of a hinged-slide joint, whereby it loses balance in the initial position [16]. In this study, efforts were made to minimize the buckling problem by supporting the segment using flat springs at the initial position. The prediction model introduced in this study expresses the overall position error as the accumulated sum of the errors of each joint. Despite efforts to increase sophistication in the manufacturing process, errors still occurred. More efforts are needed to reduce mechanical errors by designing an assembly method that can improve precision, for example, by manufacturing a jig for fixing the position. In addition, the issue of plastic deformation of the flat spring could be avoided by replacing stainless steel with a hyper-elastic material such as nitinol. A hyper-elastic material would contribute significantly towards improving the performance of this machine, owing to its excellent flexibility and resilience. However, nitinol is more expensive than stainless steel, and substantial expertise is required for shape forming. Therefore, for this prototype production, a relatively widely used material was used. Another error occurred in the process of obtaining the stiffnesses through linearization, since linearization inherently involves some errors. Finally, modeling techniques considering friction of segments, flat springs, and cables can also aid accurate analyses. Nevertheless, it was a reasonable method to bring the stiffnesses within the shape prediction, and several repeated experiments were implemented to increase the reliability of the linearization.

\section{Conclusions}

In this study, we proposed a model that could predict the bending shape of a hyperredundant manipulator with variable non-uniform curvature. In this manipulator, flat springs with different thicknesses were arranged at each joint, and the bending shape could also be changed by changing the arrangement order through reassembly. The verification was carried out through a predictive model established by applying the minimum potential energy theory. The developed manipulator has a few limitations. An important step before it is used as a medical device is obtaining certification. The material used in this development is harmless to the human body, and it is also versatile. However, to adjust the non-uniform curvature and make the bending shape as desired, there is a cumbersome requirement to reassemble the manipulator. An innovative idea of changing the bending shape without reassembly would speed up the application time in the actual operating room. Although we require more research to improve upon our study, we believe that this could potentially be widely used in various fields. It may potentially be developed as a primary bending mechanism of an endoscope system, a single-port robot system, or a bending mechanism of a surgical tool used with a general endoscope. Owing to its small diameter, it is easy to use for surgeries in narrow spaces, such as the nasal cavity, oral cavity, or pituitary gland. In the future, this study could be further developed through inverse configuration of the predetermined environment to obtain the joints of the manipulator for more pragmatic usages in operations and incorporating monolithic design to eliminate assembling errors.

Author Contributions: Conceptualization, S.K. and J.K.; methodology, S.K. and J.K.; software, Y.M.; validation, S.K.; formal analysis, S.K.; investigation, S.K.; writing—original draft preparation, S.K.; writing - review and editing, S.K.; project administration, K.K.; funding acquisition, K.K. All authors have read and agreed to the published version of the manuscript.

Funding: This research was supported by the Technology Innovation Program (20000843, Development of fluorescence image guided transoral and laparoscopic single port surgery robot with flexibly articulated robotic instruments) funded by the Ministry of Trade, Industry \& Energy (MOTIE, Korea).

Institutional Review Board Statement: Not applicable.

Informed Consent Statement: Not applicable. 
Data Availability Statement: The data presented in this study are available on request from the first author.

Conflicts of Interest: The authors declare no conflict of interest.

\section{References}

1. Valentina, V.; Su-Lin, L.; Thomas, P.C.; Guang-Zhong, Y. Emerging robotic platforms for minimally invasive surgery. IEEE Rev. Biomed. Eng. 2013, 6, 111-126.

2. Filip, J.; Ewout, A.A.; Paul, W.J.H.; Rob, P.; Paul, B. Classification of joints used in steerable instruments for minimally invasive surgery-A review of the state of the art. J. Med. Device 2015, 9, 010801.

3. Brain, S.P.; Priscila, R.A.; Crystal, K.; Songita, A.C.; Dmitry, O. Review of emerging surgical robotic technology. Surg. Endosc. 2018, 32, 1636-1655.

4. Xiaohua, H.; Ang, C.; Yigang, L.; Chris, Z.; Edwin, Z. Steerable catheters for minimally invasive surgery: A review and future directions. Comput. Assist. Surg. 2018, 23, 21-41.

5. Pierre, B.; Konrad, L.; Kiyoung, K.; Carlo, A.S.; Jianzhong, S.; Guang-Zhong, Y. Rolling-joint design optimization for tendon driven snake-like surgical robots. In Proceedings of the IEEE/RSJ International Conference on Intelligent Robots and Systems (IROS), Madrid, Spain, 1-5 October 2018; pp. 4964-4971.

6. Daniel, J.A.; Chris, B.; Richard, I.R.; William, J.P. Design of an endoluminal NOTES robotic system. In Proceedings of the IEEE/RSJ International Conference on Intelligent Robots and Systems (IROS), San Diego, CA, USA, 29 October-2 November 2007; pp. 410-416.

7. Ben Brown, H.; Michael, S.; Shammas, E.; Choset, H. Design and control of a second-generation hyper-redundant mechanism. In Proceedings of the IEEE/RSJ International Conference on Intelligent Robots and Systems (IROS), San Diego, CA, USA, 29 October-2 November 2007; pp. 2603-2608.

8. Arjo, L.; Paul, B.; Jenny, D. Scopes too flexible and too stiff. IEEE Pulse 2010, 1, $26-41$.

9. Hossein, D.; Shane, F.; Dmitry, O.; Benjamin, T. Automation of Suturing Path Generation for da Vinci-Like Surgical Robotic Systems. In Proceedings of the Design of Medical Devices Conference (DMD), Minneapolis, MN, USA, 9-12 April 2018.

10. Sahba, A.P.; Changyeob, S.; Peter, W.F.; Ji, M.; Erik, P.D.; Jacob, R. Autonomous Suturing Framework and Quantification Using a Cable-Driven Surgical Robot. IEEE Trans. Robot. 2021, 37, 404-417.

11. Changyeob, S.; Peter, W.F.; Sahba, A.P.; Ji, M.; Erik, P.D.; Jacob, R. Autonomous Tissue Manipulation via Surgical Robot Using Learning Based Model Predictive Control. In Proceedings of the International Conference on Robotics and Automation (ICRA), Montreal, QU, Canada, 20-24 May 2019; pp. 3875-3881.

12. Robert, J.W.; Joseph, M.R.; Noah, J.C. Mechanics of precurved-tube continuum robots. IEEE Trans. Robot. 2009, $25,67-78$.

13. Mohsen, M.D.; Saeid, N.; Robert, D.H. An Analytical Loading Model for $n$-Tendon Continuum Robots. IEEE Trans. Robot. 2018, $34,1215-1225$.

14. Tobias, M.; Alexander, H.; Oliver, S. A variable curvature continuum kinematics for kinematic control of the bionic handling assistant. IEEE Trans. Robot. 2014, 30, 935-949.

15. Kiyoung, K.; Hynsoo, W.; Jungwook, S. Design and evaluation of a continuum robot with discreted link joints for cardiovascular interventions. In Proceedings of the IEEE International Conference on Biomedical Robotics and Biomechatronics (Biorob), Enschede, The Netherlands, 26-29 August 2018; pp. 627-633.

16. Jeongryul, K.; Yonghwan, M.; Seong-il, K.; Keri, K. Accurate estimation of the position and shape of the rolling joint in hyperredundant manipulators. In Proceedings of the IEEE/RSJ International Conference on Intelligent Robots and Systems (IROS), Las Vegas, NV, USA, 25-29 October 2020; pp. 3055-3060.

17. Suh, J.W.; Kim, K.Y.; Jeong, J.W.; Lee, J.J. Design considerations for a hyper-redundant pulleyless rolling joint with elastic fixtures. IEEE/ASME Trans. Mechatron. 2015, 20, 2841-2852. [CrossRef]

18. Yong-jae, K.; Shanbao, C.; Sangbae, K.; Karl, I. A stiffness-adjustable hyperredundant manipulator using a variable neutral-line mechanism for minimally invasive surgery. IEEE Trans. Robotics 2013, 30, 382-395.

19. Minho, H.; Dong-Soo, K. K-FLEX: A flexible robotic platform for scar-free endoscopic surgery. Int. J. Med. Robot 2020, 16, e2078.

20. Rucker, D.C. and Webster, R.J., III.Statics and Dynamics of continuum Robots with General Tendon Routing and External Loading. IEEE Trans. Robot. 2011, 27, 1033-1044. [CrossRef]

21. Yeongjin, K.; Shing, S.C.; Jaydev, P.D. Active stiffness tuning of a spring-based continuum robot for MRI-guided neurosurgery. IEEE Trans. Robot. 2017, 34, 18-28.

22. Yeongjin, K.; Shing, S.C.; Mahamadou, D.; Rao, P.G.; Simard, J.M.; Jaydev, P.D. Toward the development of a flexible mesoscale MRI-compatible neurosurgical continuum robot. IEEE Trans. Robot. 2017, 33, 1386-1397.

23. Fei, J.; Haijie, Z.; Jianguo, Z. Kinematics and statics for soft continuum manipulators with heterogeneous soft materials. In Proceedings of the Dynamic Systems and Control Conference, Minneapolis, MN, USA, 12-14 October 2016 ; p. 50701.

24. Long, H.; Bei, L.; Lairong, Y.; Peng, Z.; Yuanhan, Y. Design and Validation of a Novel Cable-Driven Hyper-Redundant Robot Based on Decoupled Joints. Hindawi J. Robot. 2021, 2021, 5124816.

25. Guodong, Q.; Aihong, J.; Yong, C.; Wenlong, Z.; Hongtao, P.; Shangshuang, S.; Yuntao, S. A Snake-Inspired Layer-Driven Continuum Robot. Soft Robot. 2021. ahead of print. [CrossRef] 
26. Zheng, L.; Haoyong, Y.; Hongliang, R.; Philip, W.Y.C.; Ruxu, D. A Novel Constrained Tendon-driven Serpentine Manipulator. In Proceedings of the IEEE/RSJ International Conference on Intelligent Robots and Systems (IROS), Hamburga, Germany, 28 September-2 October 2015; pp. 5966-5971.

27. Cristina, P.; Cristian, V.; Daniela, P.; Florina, B.; Stefan, C.; Andrei, T.; Ionut, R.; Nicu, B. Position control for hybrid infinitecontinuous hyper-redundant robot. MATEC Int. Conf. Manuf. Sci. Educ. 2021, 343, 08009.

28. Mingfeng, W.; Xin, D.; Weiming, B.; Abdelkhalick, M.; Dragos, A.; Andy, N. Design, modelling and validation of a novel extra slender continuum robot for in-situ inspection and repair in aeroengine. ELSEVIER Robot. Comput. Integr. Manuf. 2021, 67, 102054.

29. Matteo, R.; Luca, R.; Xin, D.; Dragos, A.; James, K. Task-oriented optimal dimensional synthesis of robotic manipulators with limited mobility. ELSEVIER Robot. Comput. Integr. Manuf. 2021, 67, 102096.

30. Seriani, S.; Gallina, P.; Scalera, L.; Lughi, V. Development of $n$-DoF Preloaded Structures for Impact Mitigation in Cobots. J. Mech. Robot. 2018, 10, 051009. [CrossRef] 